aortic velocity $3.1 \pm 0.6 / \mathrm{ms}$, LV mass $172 \pm 48 \mathrm{~g}$; SBP $127 \pm 14 \mathrm{~mm}$ $\mathrm{Hg}$ DBP $76 \pm 10 \mathrm{~mm} \mathrm{Hg}$ ) and 18 patients with velocity-matched TAV (age $74 \pm 6$ years, female $28 \%$, velocity $3.1 \pm 0.6 / \mathrm{ms}$, LV mass $147 \pm 27 \mathrm{~g}$; SBP $136 \pm 17 \mathrm{~mm} \mathrm{Hg}$ DBP $79 \pm 7 \mathrm{~mm} \mathrm{Hg}$; Abstract 166 table 1). Patients were scanned using a 1.5 T Avanto scanner (Siemens Healthcare, Erlangen, Germany) and basal, mid-ventricular and apical short axis tagging images were acquired. Peak systolic global circumferential strain was calculated at each ventricular level using CimTag2D software v.7 (Auckland MRI Research Group, New Zealand).

Abstract 166 Table 1 Bicuspid vs tricuspid aortic valve disease

\begin{tabular}{lccr}
\hline & $\begin{array}{l}\text { Bicuspid } \\
\text { aortic valve } \\
\text { (BAV) }\end{array}$ & $\begin{array}{l}\text { Tricuspid aortic } \\
\text { valve (TAV) }\end{array}$ & p Value \\
\hline Age (yrs) & $55 \pm 15$ & $74 \pm 6$ & $<0.01$ \\
Female (\%) & 21 & 28 & 0.31 \\
Transvalvular velocity (ms ${ }^{-1}$ ) & $3.1 \pm 0.6$ & $3.1 \pm 0.6$ & 0.50 \\
Left ventricular mass (g) & $172 \pm 48$ & $147 \pm 27$ & 0.04 \\
Systolic blood pressure (SBP) & $127 \pm 14$ & $136 \pm 17$ & 0.04 \\
Diastolic blood pressure (DBP) & $76 \pm 10$ & $79 \pm 7$ & 0.15 \\
Basal circumferential systolic strain (\%) & $20 \pm 3$ & $22 \pm 3$ & 0.04 \\
Mid ventricular circumferential strain (\%) & $19 \pm 2$ & $21 \pm 2$ & 0.07 \\
Apical ventricular circumferential strain (\%) & $17 \pm 4$ & $19 \pm 3$ & 0.04 \\
\hline
\end{tabular}

Results Patients with BAV had significantly greater left ventricular hypertrophy (BAV $172 \pm 48 \mathrm{~g}$ vs TAV $147 \pm 27 \mathrm{~g} ; \mathrm{p}=0.04$ ) despite similar degrees of valve stenosis. Peak systolic circumferential strain was lower (ie, reduced contractility) in patients with BAV than TAV (Basal BAV $20 \pm 3 \%$ vs TAV $22 \pm 3 \% \mathrm{P}=0.04$; Mid BAV $19 \pm 2 \%$ vs TAV $21 \pm 2 \% \mathrm{p}=0.07$; apical BAV $17 \pm 4 \%$ vs TAV $19 \pm 3 \%$; $<<0.04$ ), despite the younger age and lower blood pressure.

Conclusion Ventricular remodelling differs between BAV and TAV patients with equivalent transvalvular gradients. BAV patients, despite being younger and having lower systolic blood pressure, have more severe hypertrophy and lower myocardial contractility. This finding may have implications for monitoring disease progression or more timely medical or surgical intervention in patients with BAV.

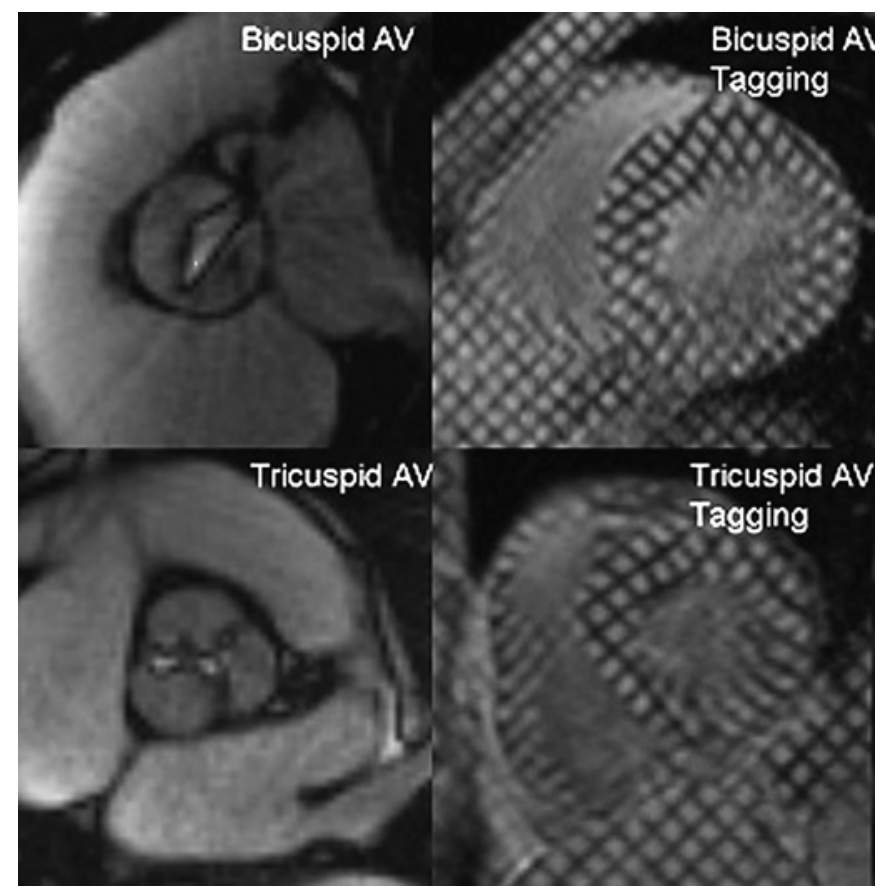

Abstract 166 Figure 1 Velocity matched tricuspid and bicuspid aortic valves showing valve morphology, tagging and cine imaging.

\section{AORTIC REGURGITATION QUANTIFICATION WITH CARDIOVASCULAR MAGNETIC RESONANCE PREDICTS CLINICAL OUTCOME}

doi:10.1136/heartjnl-2011-300198.167

${ }^{1} \mathrm{~S}$ G Myerson, ${ }^{1} \mathrm{~J}$ D'Arcy, ${ }^{2} \mathrm{R}$ Mohiaddin, ${ }^{3} \mathrm{~J} \mathrm{P}$ Greenwood, ${ }^{1} \mathrm{~T}$ D Karamitsos, 1J M Francis, 'A P Banning, ${ }^{4} \mathrm{~J}$ P Christiansen, 'S Neubauer. ${ }^{1} \mathrm{~J}$ ohn Radcliffe Hospital \& University of Oxford, Oxford, UK; ${ }^{2} \mathrm{CMR}$ Unit, Royal Brompton Hospital and the National Heart and Lung Institute, London, UK; ${ }^{3}$ University of Leeds, Leeds, UK; ${ }^{4}$ North Shore Hospital, Auckland, New Zealand

Background The timing of valve surgery in asymptomatic patients with significant aortic regurgitation can be challenging. Current indications focus on symptoms and left ventricular (LV) dilation/ dysfunction, but prognosis is already reduced by this time. Quantification of the regurgitation has not previously been used to guide management, likely due to the difficulty of achieving this with echocardiography. Cardiovascular magnetic resonance (CMR) can accurately quantify aortic regurgitation and LV volumes, and we examined whether either could predict symptom development and the need for aortic valve surgery.

Methods 94 asymptomatic patients with moderate or severe aortic regurgitation on echocardiography were identified from four high volume CMR centres. CMR scans were performed to quantify aortic regurgitation and $\mathrm{LV}$ volume indices, and subsequent clinical followup occurred for up to 7 years (mean $2.6 \pm 2.1$ years). The best predictors of progression to symptoms and other conventional indications for surgery were determined.

Results Aortic regurgitant fraction was the best predictor of clinical outcome; area under the curve (AUC) on receiver operating characteristics analysis $0.93(p<0.0001)$, with a specificity of $93 \%$ and sensitivity of $78 \%$ for predicting the progression to symptoms and surgery. Survival without surgery was $88 \%$ for patients with a regurgitant fraction $<37 \%$, compared to $6 \%$ for those with a regurgitant fraction $\geq 37 \%$ (see Abstract 167 figure 1). Regurgitant volume $>38 \mathrm{mls}$ and regurgitant volume index $>25 \mathrm{ml} / \mathrm{m}^{2}$ were also good predictors (AUC 0.91 and 0.90 respectively), though regurgitant fraction had significantly greater predictive power (OR 1.26 compared to 1.09 for regurgitant volume). LV volumetric indices also predicted outcome, but less strongly than measures of regurgitation: LV end-diastolic volume $>267 \mathrm{mls}$ (AUC 0.85), LV end-systolic volume $>88 \mathrm{mls}$ (AUC 0.78). Regurgitant fraction and volume were the only independent outcome predictors on multiple logistic regression analysis. The predictive ability of CMR applied to patients with both moderate and severe aortic regurgitation on echocardiography. Supporting data also comes from a comparison

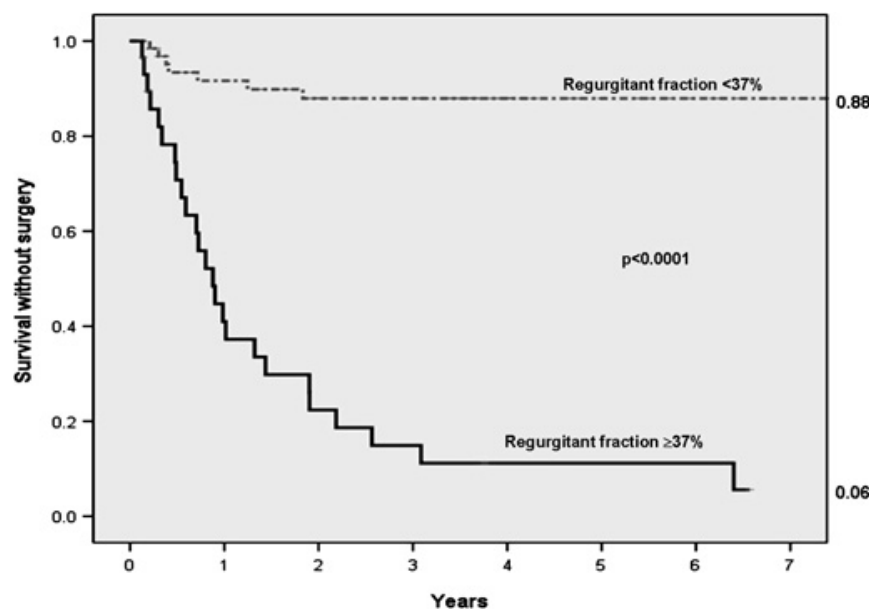

Abstract 167 Figure 1 Kaplan-Meier survival curve showing survival without surgery for conventional indications. 
with patients already planning to undergo surgery at the time of CMR scanning, which showed similar regurgitant fractions in the surgical group (mean \pm SD: $45.4 \% \pm 12.1 \%$ ) compared to the initially asymptomatic patients who developed symptoms or other indications for surgery $(42.8 \% \pm 10.4 \%) ; p=0.32$. Subjects who remained asymptomatic had a significantly lower regurgitant fraction: $25.3 \pm 8.6 \%(p<0.0001$ vs both the planned surgical group and the symptom progression group).

Conclusions CMR quantification of aortic regurgitation and LV volumes accurately predicts the progression to symptoms/surgery. Its use in patients with aortic regurgitation should be encouraged.

\section{$168 \quad \beta$-BLOCKER THERAPY IMPROVES CLINICAL OUTCOMES IN PATIENTS WITH MODERATE TO SEVERE MITRAL REGURGITATION}

\section{doi:10.1136/heartjnl-2011-300198.168}

A Nadir, D H Elder, M G MacArtney, S D Pringle, A M Choy, A D Struthers, C C Lang. University of Dundee, Dundee, UK

Background Volume overload seen in mitral regurgitation (MR) leads to neuro-endocrine activation including heightened sympathetic activity. Experimental data have reported protective effects of beta-blockers (BBs) on myocardial function in MR suggesting that $\mathrm{BB}$ therapy may be beneficial in MR. However, the effect of $\mathrm{BB}$ therapy on clinical outcomes in MR has not been defined. Hence, in this large observational study we investigated the impact of $\mathrm{BB}$ therapy on clinical outcome in patients with moderate to severe MR.

Methods The Health Informatics dispensed prescribing, morbidity and mortality database for the population of Tayside, Scotland was linked through a unique patient identifier to the Tayside echocardiography database (>110 000 scans). Patients with a diagnosis of moderate or severe MR from 1993 to 2008 were identified. Cox regression model (adjusted for age, gender, left ventricular dimensions and function, left atrial diameter, cardiovascular (CV) history and concurrent $\mathrm{CV}$ medications) was used to assess the impact of $\mathrm{BB}$ therapy on all-cause mortality and cardiovascular events (CV death or hospitalisations).

Results A total of 4437 patients with moderate to severe MR (mean age $74 \hat{A} \pm 11$ years, $46 \%$ males) were identified. MR was categorised as functional in $2523(57 \%)$ and organic in 1894 (43\%) while 1324 $(30 \%)$ were on BBs. Over a mean follow-up of 3.9 years there were 2287 (51\%) all-cause deaths and 2333 (52\%) CV events. Those treated with BBs had a significantly lower all-cause mortality with an adjusted HR of 0.65 (95\% CI 0.56 to $0.75, \mathrm{p}<0.0001)$ and fewer CV events with an adjusted HR of 0.79 (95\% CI 0.69 to 0.90 , $\mathrm{p}<0.0001)$

Conclusions This large observational study suggests that BB therapy is associated with an improved survival and a lower risk of CV events in patients with moderate to severe MR. These observations needs to be confirmed in prospective studies and support the rationale for undertaking a future randomised clinical trial.

\section{MID-WALL FIBROSIS IS AN INDEPENDENT PREDICTOR OF MORTALITY IN PATIENTS WITH AORTIC STENOSIS}

doi:10.1136/heartjnl-2011-300198.169

\footnotetext{
1,2M R Dweck, ${ }^{1} \mathrm{~S}$ Joshi, ${ }^{1} \mathrm{~T}$ Murigu, ${ }^{1} \mathrm{~A}$ Gulati, ${ }^{1} \mathrm{~F}$ Alpendurado, ${ }^{1} \mathrm{R}$ Mohiaddin, ${ }^{1} \mathrm{~J}$ Pepper, ${ }^{1} \mathrm{D}$ Pennell, ${ }^{2} \mathrm{D}$ Newby, ${ }^{1} \mathrm{~S}$ Prasad. ${ }^{1}$ Royal Brompton Hospital, London, UK; ${ }^{2}$ Centre for Cardiovascular Sciences, University of Edinburgh, Edinburgh, UK
}

Introduction Predicting adverse clinical outcomes in aortic stenosis is challenging. Late gadolinium enhancement (LGE) has been asso- ciated with an adverse prognosis in a range of other cardiac conditions. Using late gadolinium enhancement, we sought to assess the prognostic significance of mid-wall and infarct patterns of myocardial fibrosis in aortic stenosis.

Methods Between January 2003 and October 2008, consecutive patients with moderate or severe aortic stenosis (aortic valve area $<1.5 \mathrm{~cm}^{2}$ ) underwent cardiovascular magnetic resonance with assessment of myocardial fibrosis by late gadolinium enhancement. Patients were categorised into absent, mid-wall or infarct patterns of late gadolinium enhancement by blinded independent observers. Patient follow-up was completed using the National Strategic Tracing Scheme.

Results 143 patients (aged 68 \pm 14 years; 97 male) were followed up for $2.0 \pm 1.4$ years. 81 patients had coronary artery disease, 72 underwent aortic valve replacement and 27 died. Compared to those with no late gadolinium enhancement $(n=49)$, univariate analysis revealed that patients with mid-wall fibrosis $(n=54)$ had an eightfold increase in all-cause mortality despite similar aortic stenosis severity and coronary artery disease burden. Patients with an infarct pattern $(n=40)$ had a six-fold increase. Mid-wall fibrosis (HR, 5.35 (95\% CI, 1.16 to 24.56); $p=0.03$ ) and ejection fraction (HR 0.96 (95\% CI, 0.94 to 0.99 ); $p=0.01$ ) were independent predictors of all cause mortality by multivariate analysis. Conclusion: Mid-wall fibrosis is an independent predictor of mortality in patients with moderate and severe aortic stenosis. It has incremental prognostic value to ejection fraction and may provide a useful method of risk stratification in patients with advanced disease (Abstract 169 figure 1).

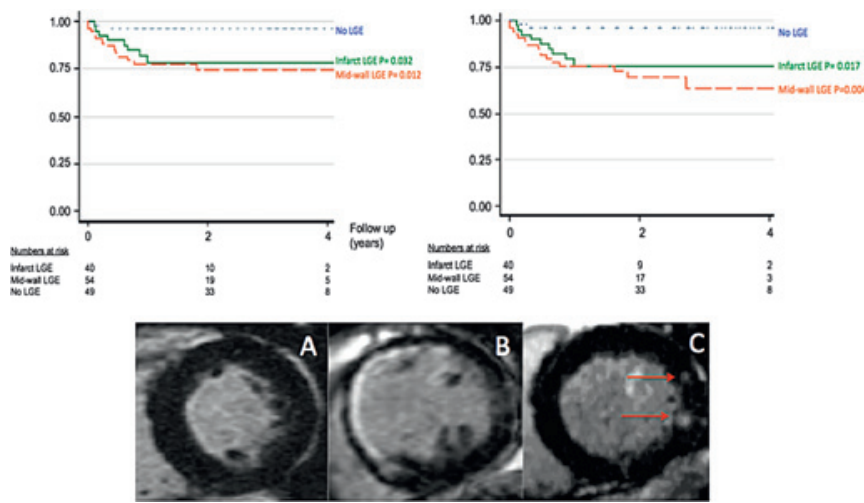

Abstract 169 Figure 1 Kaplan-Meier curves of cardiac mortality (left) and all cause mortality (right) according to pattern of $L G E(A=$ No $L G E$, $\mathrm{B}=$ Infarct $\mathrm{LGE}, \mathrm{C}=$ Mid-wall $\mathrm{LGE}$ ).

Abstract 169 Table 1

\begin{tabular}{lcclr}
\hline & No LGE & Mid-wall LGE & Infarct LGE & p Value \\
\hline Number of patients & 49 & 54 & 40 & - \\
Mean age yrs & $64 \pm 16$ & $70 \pm 11$ & $70 \pm 13$ & 0.031 \\
Documented CAD \% & 37 & 42 & 98 & $<0.001$ \\
Ejection fraction \% & $69 \pm 13$ & $58 \pm 21$ & $44 \pm 18$ & $<0.001$ \\
Aortic valve area & $1.05 \pm 0.37$ & $1.00 \pm 0.31$ & $0.91 \pm 0.26$ & 0.111 \\
Indexed LV mass g/m2 & $92.6^{*}$ & $113.7^{*}$ & $97.8^{*}$ & 0.005 \\
& $(86.0,99.6)$ & $(104.5,123.8)$ & $(90.9,105.2)$ & \\
Mortality rate (deaths / & 15.7 & 142.7 & 173.7 & \\
1000 pt years) & & & & \\
& $*$ Geometric & & & \\
& mean (95\%) & & & \\
& & & & \\
& & & &
\end{tabular}

